\title{
UCRL-CONF-217796
}

LAW RENCE LIVERMORE N A T IO N A L LABORATORY

\section{Detection of Laser Optic Defects Using Gradient Direction Matching}

B. Y. Chen, L. M. Kegelmeyer, J. A. Liebman, J.

T. Salmon, J. Tzeng, D. W. Paglieroni

December 19, 2005

Photonics West 2006

San Jose, CA, United States

January 21, 2006 through January 26, 2006 
This document was prepared as an account of work sponsored by an agency of the United States Government. Neither the United States Government nor the University of California nor any of their employees, makes any warranty, express or implied, or assumes any legal liability or responsibility for the accuracy, completeness, or usefulness of any information, apparatus, product, or process disclosed, or represents that its use would not infringe privately owned rights. Reference herein to any specific commercial product, process, or service by trade name, trademark, manufacturer, or otherwise, does not necessarily constitute or imply its endorsement, recommendation, or favoring by the United States Government or the University of California. The views and opinions of authors expressed herein do not necessarily state or reflect those of the United States Government or the University of California, and shall not be used for advertising or product endorsement purposes. 


\title{
Detection of Laser Optic Defects Using Gradient Direction Matching ${ }^{1}$
}

\author{
Barry Y. Chen*, Laura M. Kegelmeyer, Judith A. Liebman, J. Thaddeus Salmon, Jack Tzeng, and \\ David W. Paglieroni \\ Lawrence Livermore National Laboratory, 7000 East Avenue, Livermore, CA 94550 \\ UCRL-CONF-217796
}

\begin{abstract}
That National Ignition Facility (NIF) at Lawrence Livermore National Laboratory (LLNL) will be the world's largest and most energetic laser. It has thousands of optics and depends heavily on the quality and performance of these optics. Over the past several years, we have developed the NIF Optics Inspection Analysis System that automatically finds defects in a specific optic by analyzing images taken of that optic.

This paper describes a new and complementary approach for the automatic detection of defects based on detecting the diffraction ring patterns in downstream optic images caused by defects in upstream optics. Our approach applies a robust pattern matching algorithm for images called Gradient Direction Matching (GDM). GDM compares the gradient directions (the direction of flow from dark to light) of pixels in a test image to those of a specified model and identifies regions in the test image whose gradient directions are most in line with those of the specified model. For finding rings, we use luminance disk models whose pixels have gradient directions all pointing toward the center of the disk. After GDM identifies potential rings locations, we rank these rings by how well they fit the theoretical diffraction ring pattern equation. We perform false alarm mitigation by throwing out rings of low fit. A byproduct of this fitting procedure is an estimate of the size of the defect and its distance from the image plane. We demonstrate the potential effectiveness of this approach by showing examples of rings detected in real images of NIF optics.
\end{abstract}

\section{INTRODUCTION}

The National Ignition Facility (NIF) at Lawrence Livermore National Laboratory (LLNL) is a research facility for ignition and high energy density physics. When completed, NIF will have thousands of optics that guide, amplify, and tightly focus the light from 192 laser beamlines onto a tiny target. Camera systems throughout the laser take pictures of individual optics for an automatic optics inspection system. The goal of this system is to automatically detect and characterize defects in optics over time, providing information for deciding whether to repair, replace, or continue safely using an optic.

Custom algorithms, developed at LLNL for NIF detect and characterize defects on specific optics using a "direct" approach that finds evidence of these flaws in images of the optics on which they reside [1]. These algorithms also estimate a goodness score for each detected flaw representing the system's confidence in the detection. For the most part, these algorithms work, yet in some situations the automatic detection of flaws is hindered by noise, ghost reflections, and other optical effects. Until now, the detection algorithm and the goodness score estimator have utilized only information coming from images of the optics on which the flaws reside.

Another approach for detecting defects in upstream optics seeks to find indirect evidence of defects in the form of the diffraction ring patterns on images of downstream optics. The presence of rings in a downstream optic image is evidence that a defect exists in some upstream optic. This "indirect" approach can complement the previously developed "direct" approach by helping to detect flaws that were missed by the "direct" approach and by offering a new source of information to improve the goodness estimate. As an example of the latter, consider flaws detected by the "direct" algorithm which do not lead to rings in downstream optics. These are likely false alarms, and a system that uses ring detection information would be able to significantly lower the goodness scores of these false alarms.

Diffraction ring patterns have been used in the past to characterize the size and shape of the objects that cause them. They have been used to infer size and shape information of fine particles [2], and for gauging the thicknesses of wires [3]. We are primarily interested in using diffraction rings to detect the presence of a defect in an optic and estimate its size as well as identify on which upstream optic the defect resides.

This paper describes our approach for automatically detecting diffraction ring patterns in images of optics. Section 2 describes the physical process that creates diffraction rings as well as our prior attempts at developing

\footnotetext{
${ }^{1}$ This work was performed under the auspices of the U.S. Department of Energy by the University of California, Lawrence Livermore National Laboratory under Contract No. W-7405-Eng-48.

* Corresponding author - email: chen52@,1lnl.gov
} 
programs for automatically detecting the rings. Section 3 describes all the components of our new approach for finding rings using a robust pattern matching tool called Gradient Direction Matching (GDM) [4, 5]. Section 4 shows and discusses the results of our algorithm for finding rings, and finally Section 5 summarizes our findings and outlines future work.

\section{Diffraction Ring Patterns}

The primary mechanism for the production of diffraction ring patterns is depicted in Figure 1. These rings appear as a result of the interference between the laser plane wave and a spherical wave created by a defect modeled as a circular opaque scattering source. The ring intensity $I(r, R, z)$ of an image pixel at a distance $r$ from the center of the ring pattern is a function of the defect radius $R$ and the distance $z$ between the defect and the downstream optic and is given by equation 1 :

1) $I(r, R, z)=$

$$
\frac{r^{2}+R \cdot \text { BesselJ }\left[1, \frac{2 \pi r R}{l a m b d a \cdot z}\right]\left(R \cdot \text { BesselJ }\left[1, \frac{2 \pi r R}{l a m b d a \cdot z}\right]-2 r \sin \left[\frac{\pi r^{2}}{l a m b d a \cdot z}\right]\right)}{r^{2}}
$$

where BesselJ[1,x] is a Bessel function of the first kind, and lambda is the wavelength of the illuminating light. In general the larger the defect radius $R$, the higher the intensity amplitude of the resulting rings, and the larger the distance $z$, the larger the rings. An example of a diffraction ring pattern is pictured in the left image of Figure 3.

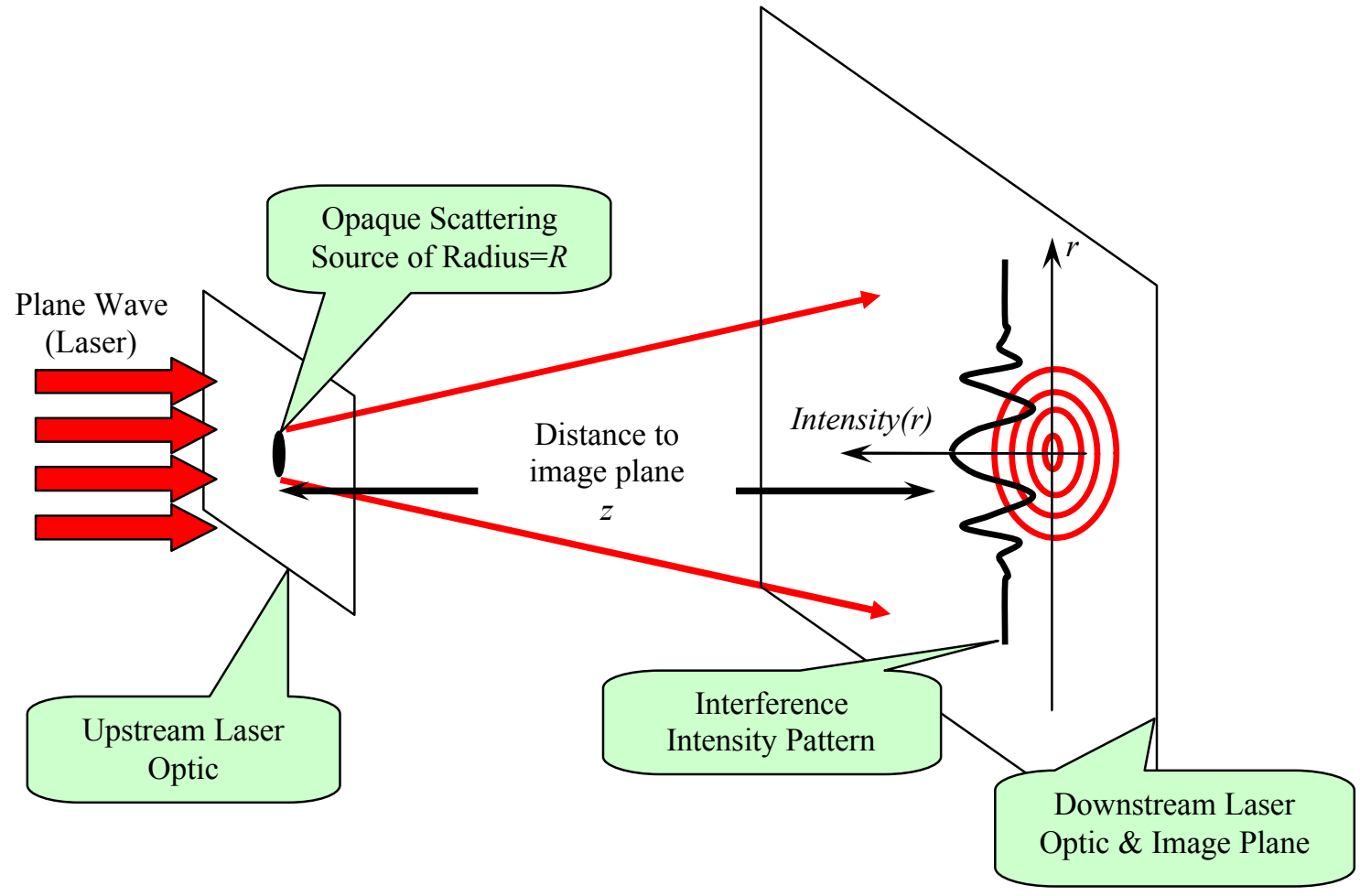

Figure 1 - Diffraction ring patterns arise from the interference between a plane wave and an opaque scattering source, e.g., defect scattering site.

\subsection{Initial Techniques for Finding Rings}

We explored using classical matched filters and variations on classical matched filters for finding diffraction ring patterns. In classical matched filtering both complex amplitude and phase are used to correlate the image to a reference pattern. Since phase information in matched filters is typically more important than amplitude information, phase-only filters [6] often give improved performance over the classical matched filter. Another variant, the amplitude 
modulated phase-only filter [7], further enhances the performance of the phase-only filter. We experimented using each of these filters with several reference patterns including perfectly circular rings of various thicknesses (corresponding to different oscillation frequencies) and sizes, and simulated diffraction patterns which were created using Equation 1. Each reference pattern and filter combination detected only ring patterns that were nearly identical to the reference pattern in size and oscillation frequency. We also applied Gaussian derivative filters, which have been effective in the past for detecting edges and curves [8,9], to detect rings, but this approach suffered from the same weakness of the matched filtering approaches - it could not robustly detect rings much different in size and frequency than the specified model. Using these techniques in our inspection system would be computationally expensive because rings in optics images come in so many different sizes and oscillation frequencies. Each combination of size and oscillation frequency would require its own reference pattern. Our new technique using Gradient Direction Matching, does not require such specific models, only the approximate size of the rings we want to detect.

\section{Detecting Diffraction Ring Patterns Using Gradient Direction Matching}

Gradient Direction Matching (GDM) is a fast and robust algorithm for finding patterns in images [4, 5]. Unlike traditional template matching methods that seek correlations in pixel intensities, GDM uses a novel measure to match pixel gradient directions (i.e., the direction of flow from dark to light) of templates to images. Gradient directions are well correlated (or give a high match value) whenever they line up, i.e., point in the same or opposite directions; whereas, orthogonal gradient directions result in low match values. The reliance on gradient directions makes GDM relatively insensitive to brightness and contrast, and variations in other image acquisition conditions. In this section we describe in more detail our approach for finding rings and how we apply GDM for detecting diffraction ring patterns. Figure 2 shows a flow diagram of our approach, which consists of several stages. The first stage uses GDM to find areas in the image most resembling the input reference models (in this case, they are disks of two different sizes). The second step identifies those areas with highest match similarity values, followed by a third stage that finds the local maxima within these areas and outputs an initial set of ring centers. The final stage is a false alarm mitigation step that measures the goodness of fit of the detected rings to Equation 1 and discards poor fits. The output is a set of ring locations and defect size and distance estimates.

\subsection{Stage 1: Gradient Direction Matching}

In the first stage, GDM is used to find areas in the image most resembling the input reference model or template. GDM first computes the gradient directions of the pixels in the test image and the reference pattern image. The computation of gradient directions in GDM is quite flexible allowing the user to specify the size of the neighborhood of pixels over which to compute the gradient direction. In this work, the gradient directions computed by GDM are identical to ones obtained using $3 \times 3$ Sobel operators [10]. Figure 3 shows an image of an example diffraction ring pattern found in our images and its corresponding gradient direction flow field. The gradient directions of ring pixels tend to flow either toward the center of the ring or away from the center of the ring. In traditional template matching approaches, one would need to specify a template reflecting not only the ring size, but also the distance from one concentric ring to the next. For detecting rings using GDM, we merely specify a template that has gradient directions all flowing toward the center of a circle since these directions will give high match values with the inward and outward pointing gradient directions found in diffraction ring patterns. A template that has this property is the luminance disk model whose pixel intensities increase linearly with decreasing distance to the center. Figure 4 shows this luminance disk model and its corresponding gradient direction flow field. When using GDM to detect rings we do need to specify the radius of the luminance disk, but unlike the approaches discussed in Section 2.1, the size of rings detected by GDM is not extremely sensitive to this specification. In practice, the ring patterns detected using a luminance disk of radius $r$ are those with a radius greater than $r / 2$ and have at least one visible concentric circle with a radius less than $r$. In the flow diagram (Figure 2) there are two parallel pipelines - one dedicated to finding small rings using a luminance disk of radius 12 pixels and one for finding medium rings using a disk of radius 26 . At this point, we primarily want to detect small and medium sized rings, but our approach is flexible enough to extend to larger rings by adding additional pipelines using larger luminance disks. 


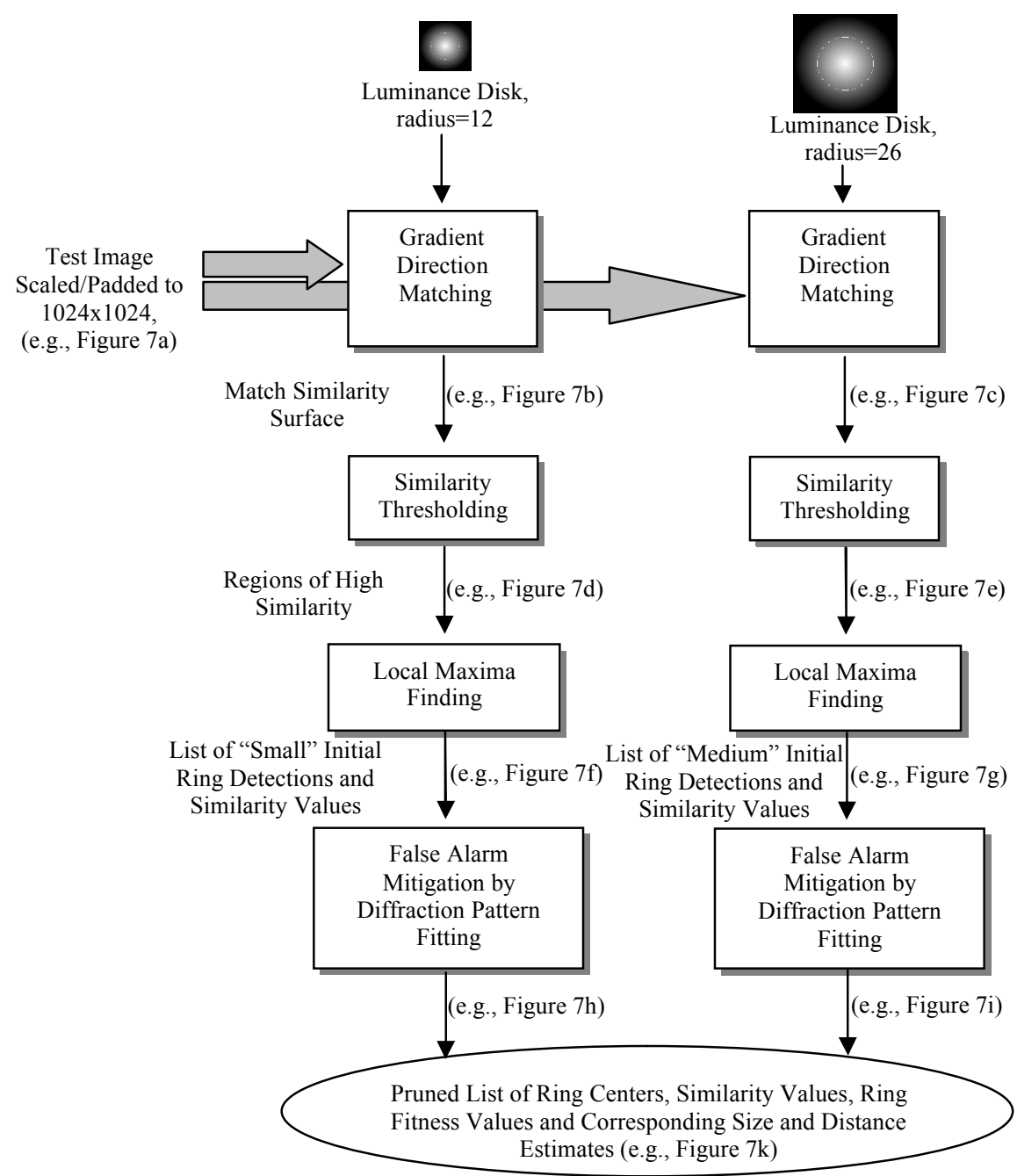

Figure 2 - Box diagram of our approach for finding diffraction ring patterns in optics.
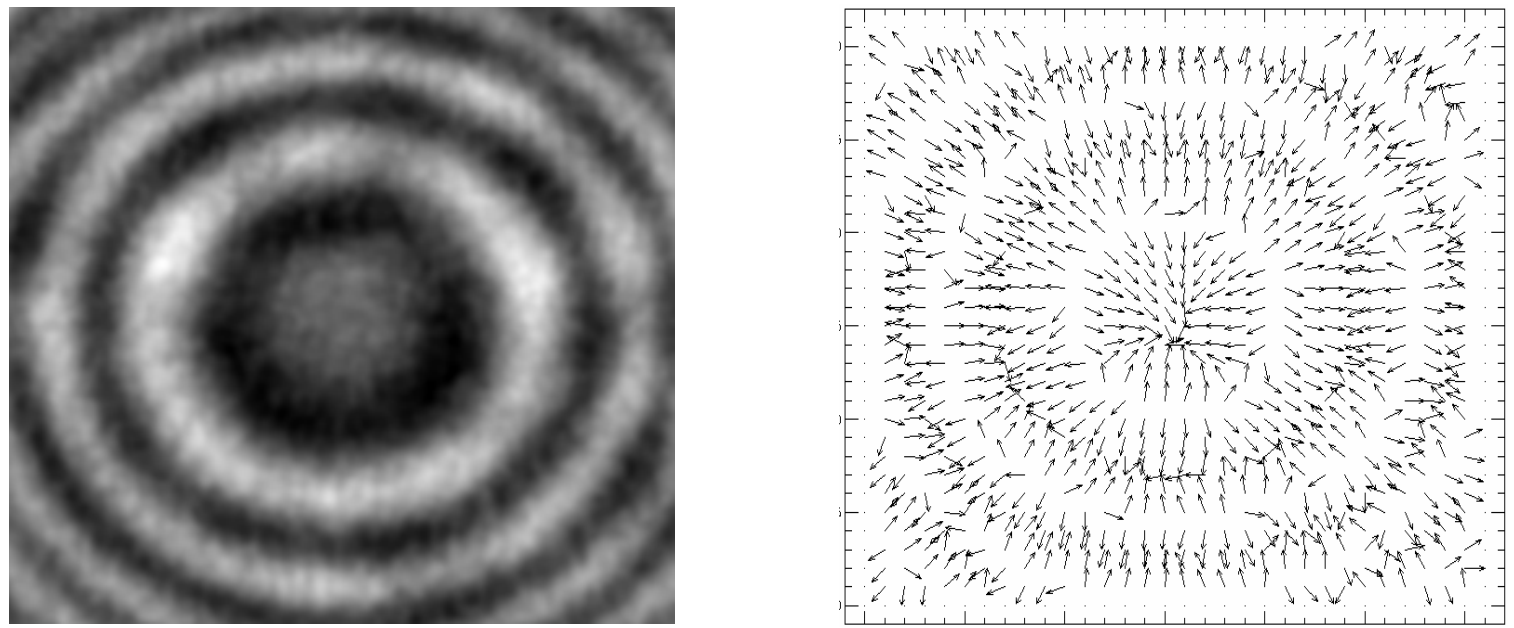

Figure 3 - Example diffraction ring pattern image and corresponding gradient direction flow field. Arrows point in the direction from dark to light. 

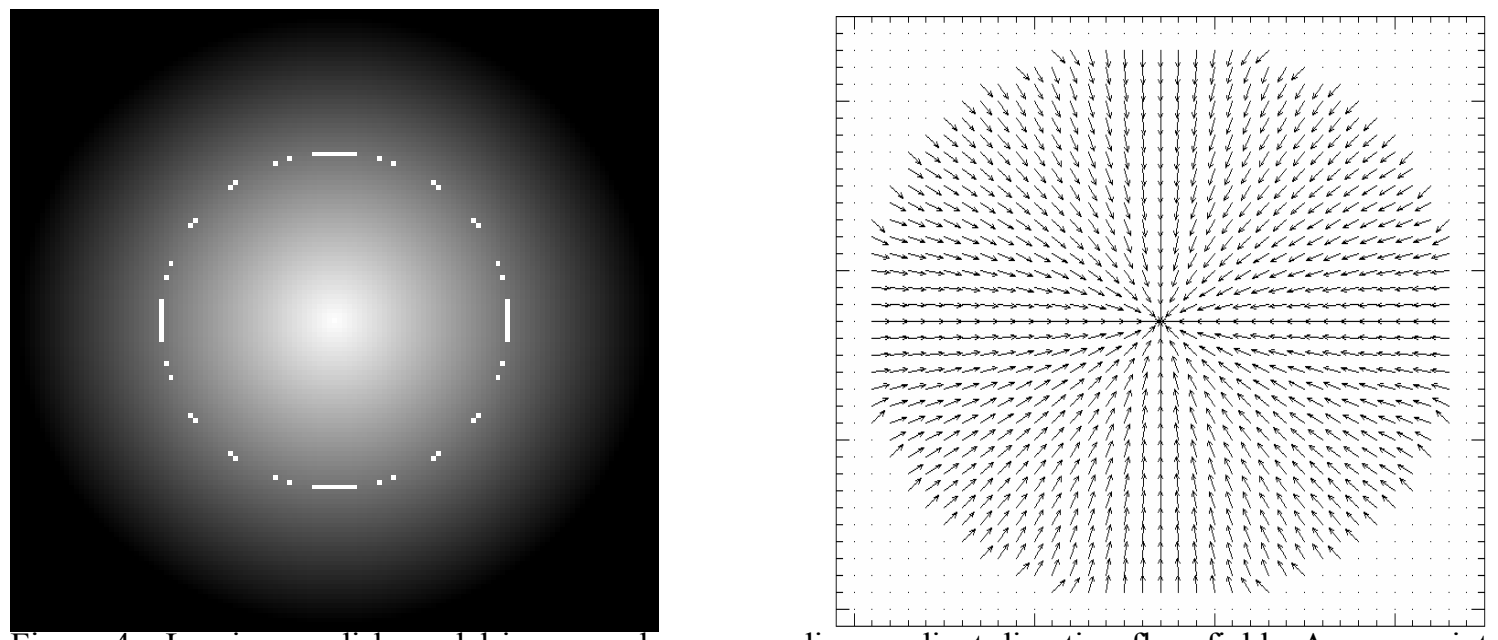

Figure 4 - Luminance disk model image and corresponding gradient direction flow field. Arrows point in the direction from dark to light.

The next step in GDM is the computation of match similarities for all pixels in the image. The match similarity at an image pixel located at $\left(c_{i}, r_{i}\right)$ is a measure of how well the model matches the image at $\left(c_{i}, r_{i}\right)$. Following $[4,5]$, the match similarity $S\left(c_{i}, r_{i}\right)$ is given by Equation 2 :

$$
S\left(c_{i}, r_{i}\right)=\frac{1}{2}+\frac{1}{2 N_{p}} \sum_{(c, r) \in P}\left[\cos ^{2}\left[\theta\left(c+c_{i}, r+r_{i}\right)-\beta(c, r)\right]-\frac{1}{2}\right]
$$

where $P$ is the set of $N_{p}$ model pixels, $\theta(c, r)$ and $\beta(c, r)$ are the gradient directions (in radians) of the image and model pixels, respectively, at column and row coordinates $(c, r)$. This match similarity ranges from 0 to 1 , and it is maximal when the gradient directions of the model are in line (parallel, not orthogonal) with those of the image region starting at $\left(c_{i}, r_{i}\right)$. Pictorially, the process of computing match similarities over an image is depicted in Figure 5 . The gradient directions of the reference pattern are shifted over the gradient directions of the test image, and the match similarity value is calculated in the region of overlap. The process of computing $S\left(c_{i}, r_{i}\right)$ at all locations can be rewritten as a convolution, at which point the circular convolution theorem of the DFT is applied, and all computations are efficiently performed in frequency domain using the FFT. Because FFTs are often radix 2, we zero pad or scale all input images to a size of $1024 \times 1024$ pixels.

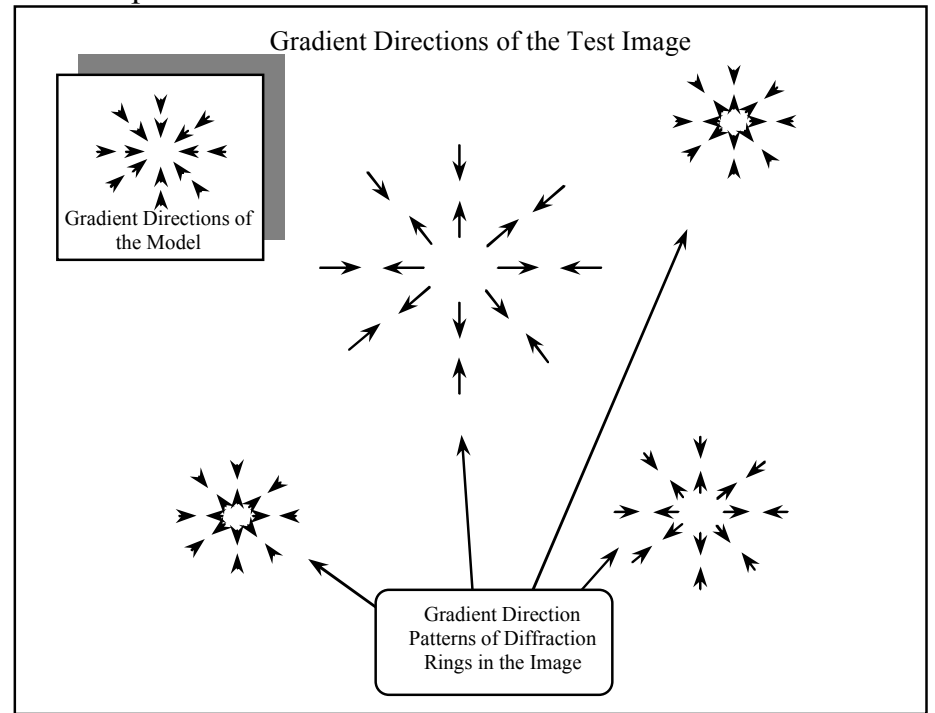

Figure 5 - GDM match similarity computation process: the match similarity between the gradient directions of the model and that of the test image are computed at every possible pixel location in the test image. 


\subsection{Stage 2 and 3: Similarity Thresholding and Local Maxima Finding}

The output of GDM is a match similarity surface consisting of match similarity values for all valid image pixels. The next step in Figure 2 is to segment out regions of high match similarity by identifying regions where the match similarity values are greater than a threshold. Thresholding in this stage acts as a filter that discards regions that are not very similar to ring patterns and ultimately controls how many rings are initially detected. The lower the threshold, the more initial rings will be detected including a significant number of false positives. We use similarity thresholds that are multiples of the standard deviation above the mode of the match similarity values. Once the regions of high similarity are identified, the algorithm outputs initial ring detections by finding the local maxima within the regions of high similarity.

\subsection{Stage 4: False Alarm Mitigation by Diffraction Ring Pattern Fitting}

The initial set of detected rings contains many false positive detections which we would like to filter out. To differentiate between false positives and real rings, the algorithm measures how well each of the initially detected rings can be described by the theoretical diffraction ring pattern in Equation 1. Starting at the detected ring centers, we compute the average intensity of pixels lying in concentric circles. A plot of these average intensities as a function of the circle radius gives us a radial intensity curve to which we can fit the theoretical diffraction ring intensity in Equation 1. Figures $6 \mathrm{a}$ and $6 \mathrm{c}$ show image regions of initially detected rings - one false alarm ring and one real ring respectively. Their corresponding radial intensity curves are pictured in Figures $6 \mathrm{~b}$ and $6 \mathrm{~d}$.

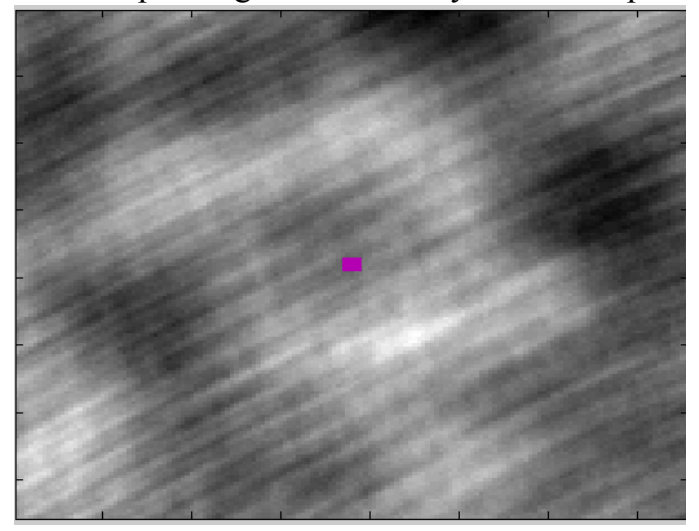

Figure $6 \mathrm{a}$ - Image region of false positive ring. Hypothesized ring center indicated by square in the middle.

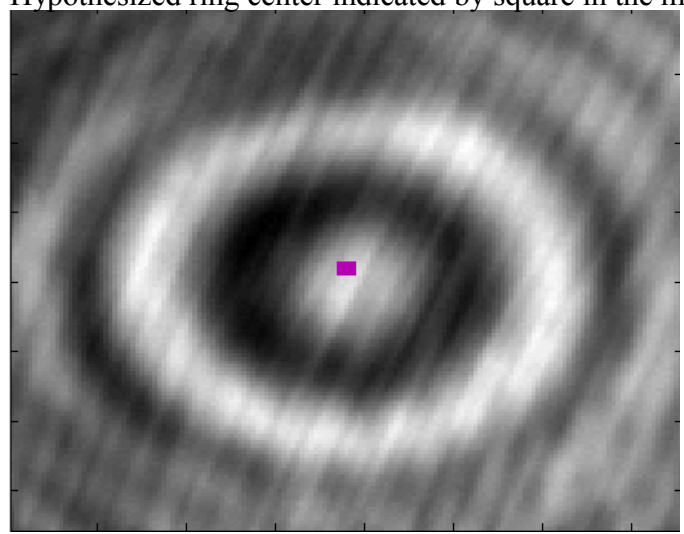

Figure $6 \mathrm{c}$ - Image region of real ring. Hypothesized ring center indicated by colored square in the middle.

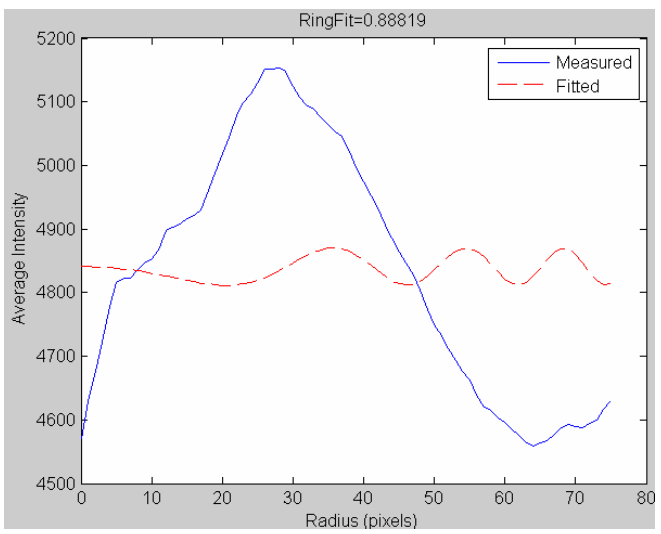

Figure $6 \mathrm{~b}$ - Measured radial intensity profile and best fit to diffraction pattern for false alarm ring.

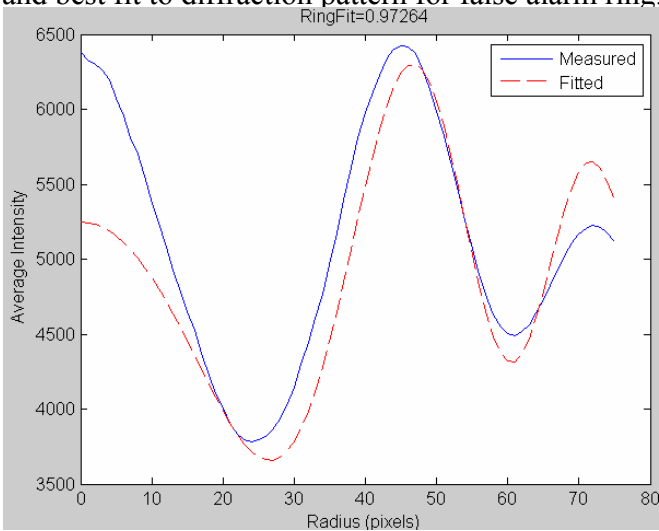

Figure 6d - Measured radial intensity profile and best fit to diffraction pattern for real ring.

We denote these measured radial intensity curves by $\bar{I}(r)$. The algorithm then finds the best fit of Equation 1 to the measured $\bar{I}(r)$ of each initial ring detected. This is done by searching over a range of values for the defect radius $R$ and the distance $z$ that minimizes the sum of squared errors (SSE) between $\bar{I}(r)$ and $I(r, R, z)$ in Equation 3 . 


$$
S S E=\sum_{r=0}^{\text {diskradius }}[I(r, R, z)-\bar{I}(r)]^{2}
$$

The sum is taken over all radii from 0 (the center pixel) to the luminance disk radius used in stage 1. SSE is a measure of how well the diffraction ring equation fits the measured radial intensity, and thus can be used as a measure of how "ringlike" an initially detected ring center is. Figures $6 \mathrm{~b}$ and $6 \mathrm{~d}$ show the resulting best fitting diffraction ring intensity curves. A byproduct of this curve fitting process is an estimate of the defect radius $R$ and the distance $z$ between the defect scattering site and the imaged optic.

In order to be able to compare the $S S E$ of different initially detected rings, it was necessary to normalize Equation 3. We first scale the optimal diffraction ring intensity curve and the measured intensity curve to lie between zero and one by subtracting the minimum intensity value (of both the ring fit curve and the measured intensity curve) and dividing by the difference between the minimum and maximum intensity value. We then divide the resulting sum squared error by the luminance disk radius. Finally, by subtracting this normalized sum squared error from 1, we get a final "ring fitness value" that ranges from zero (terrible fit) to 1 (great fit), which we can use to separate real rings from false ones. In the examples shown in Figure 6, the real ring has a ring fitness value of 0.97, while the false positive has a ring fitness value of 0.89. Almost all real rings have ring fitness values $>0.95$. In practice, the initial ring centers found in the prior stages can be slightly off center which can lead to lower ring fitness values for real rings. To compensate, the algorithm computes the ring fitness value for a set of pixels in a neighborhood of the initial ring center and returns the best ring fitness value.

\section{Processing Results on Example Images and Discussion}

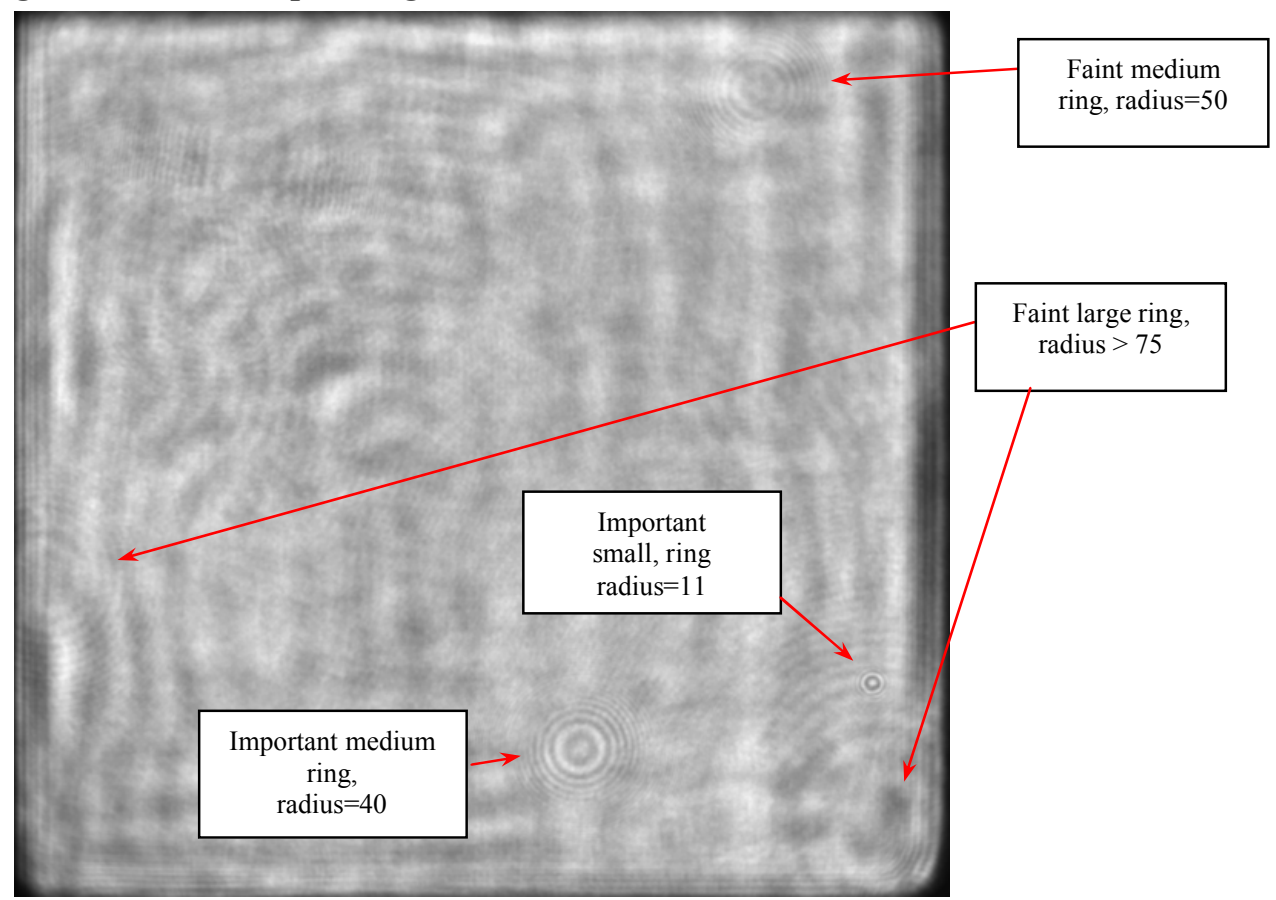

Figure 7a - Example test image 1 (744x992 pixels) with rings labeled.

In this section we walk through the processing steps in Figure 2 on a real test image. Figures $7 \mathrm{a}-7 \mathrm{~g}$ show a sample optic image with rings and the intermediate outputs after each stage in our approach. In Figure 7a, we have pointed out some rings in the original optic image. The sharp and clearly visible rings are the most important rings to detect for two reasons: first, from Equation 1, larger scattering sites lead to higher contrast rings, and second, higher contrast rings focus laser energy which can create additional scattering sites. There are two such important rings in Figure 7a, and several other unimportant, fainter and larger rings that result from reflections off of lens surfaces. In the GDM stage, we used two luminance disks - one small disk of radius 12 pixels and one medium disk of radius 26 . The match similarity surfaces computed by GDM are shown in Figures $7 \mathrm{~b}$ and $7 \mathrm{c}$. The higher the match similarity, the brighter the pixels look in the figures. The region in the image where the important small ring resides has relatively 
high similarity values when using a disk radius of 12 , but not when using a disk radius of 26 . Because the similarity value is a measure of average gradient direction correlation for all pixels in the model, when the disk radius becomes too large, the high correlation coming from pixels in the small rings will be overcome by the poor correlation of the pixels outside the small ring. The region of the important medium ring has high similarity values for both the small and medium sized disks because its center resembles a small luminance disk.

In the similarity thresholding stage, we highlight in red those regions in the match similarity surface with pixels whose values are greater than the mode plus four standard deviations as shown in Figures $7 \mathrm{~d}$ and $7 \mathrm{e}$. In Figures $7 \mathrm{f}$ and $7 \mathrm{~g}$, we have placed boxes at locations of local maxima in the high match similarity regions corresponding to the initial set of ring detections. Using the smaller disk size leads to more initial ring detections because there are many small regions that are bright in the center. All the important rings have been detected in this stage as well as the faint medium ring. The faint large rings have not been detected. Our disk radius sizes are not be large enough to find these rings.

Figures $7 \mathrm{~h}$ and $7 \mathrm{i}$, show the resulting detections after the false alarm mitigation stage where we have thrown out initial ring detections with lower ring fitness values. This stage has significantly reduced the number of false ring

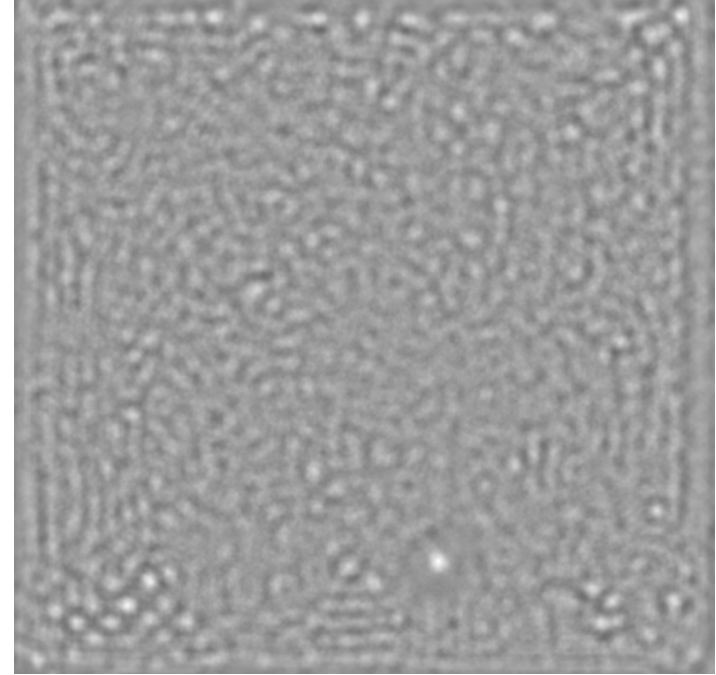

Figure $7 \mathrm{~b}$ - Match Similarity Surface for radius $=12$.

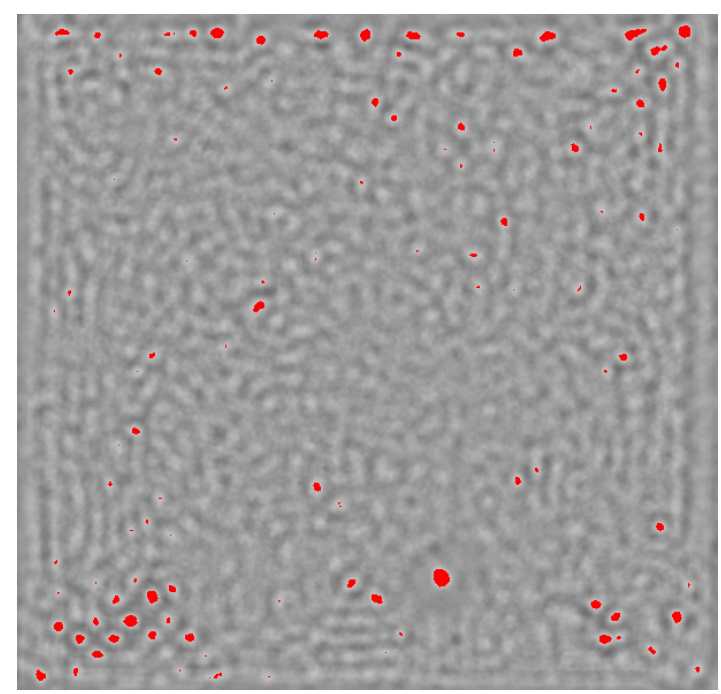

Figure $7 d$ - Regions of high similarity for radius $=12$.

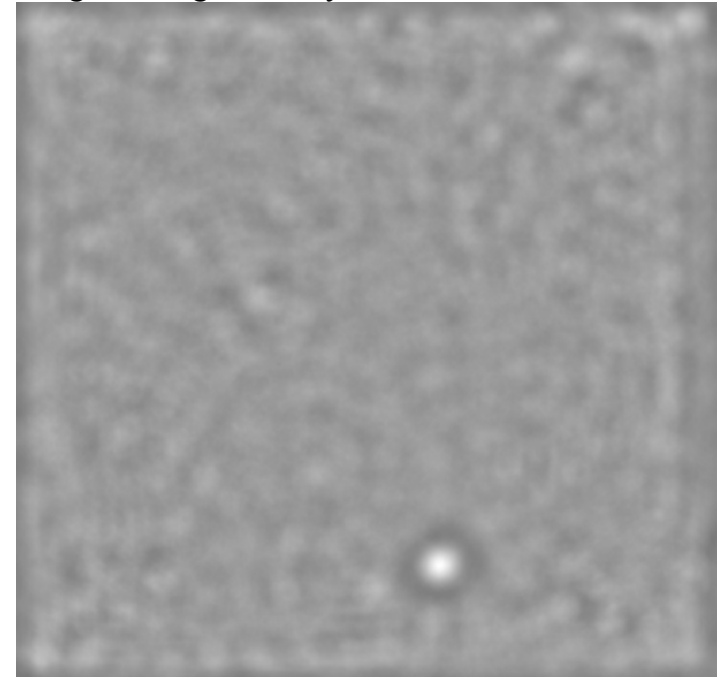

Figure 7c - Match Similarity Surface for radius $=26$.

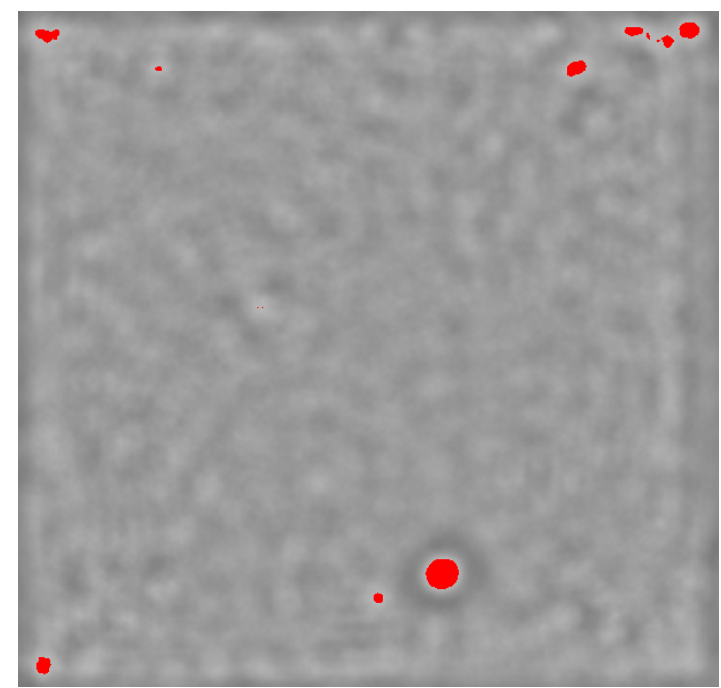

Figure $7 \mathrm{e}-$ Regions of high similarity for radius $=26$.

detections without throwing out any of the real rings detected. Figures $7 \mathrm{j}$ and $7 \mathrm{k}$ combine the detections from both small and medium disks before and after the false alarm mitigation step. Figure $7 \mathrm{k}$ shows that the algorithm has successfully detected the important rings as well as the faint medium ring with only five false alarms. 


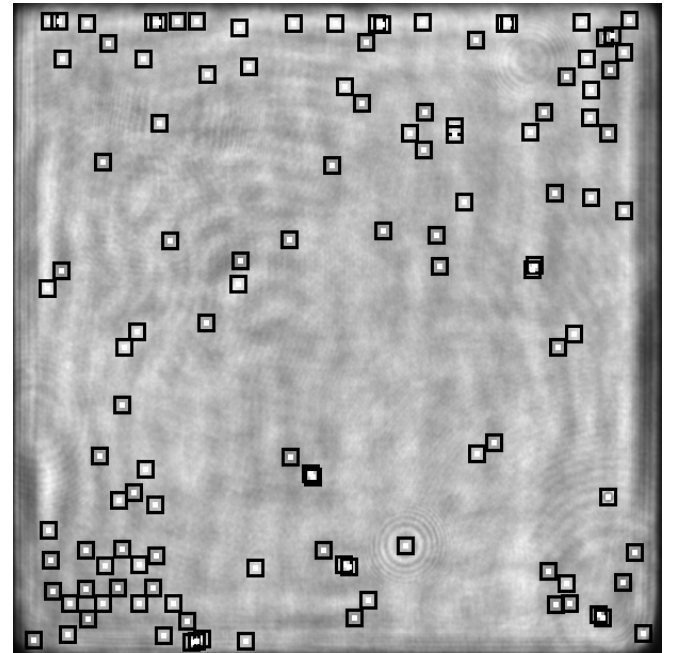

Figure $7 \mathrm{f}$ - Initial ring detections for radius $=12$.

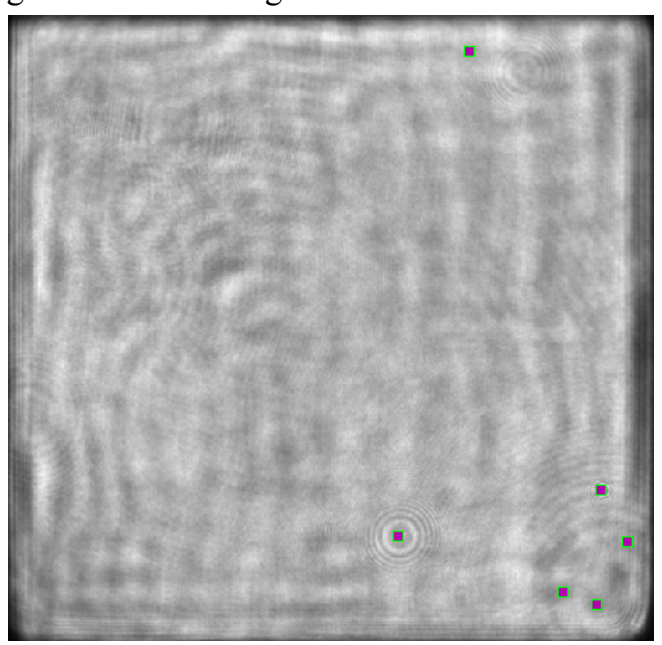

Figure $7 \mathrm{~h}$ - Ring detections (radius=12) for example test image 1 after false alarm mitigation.

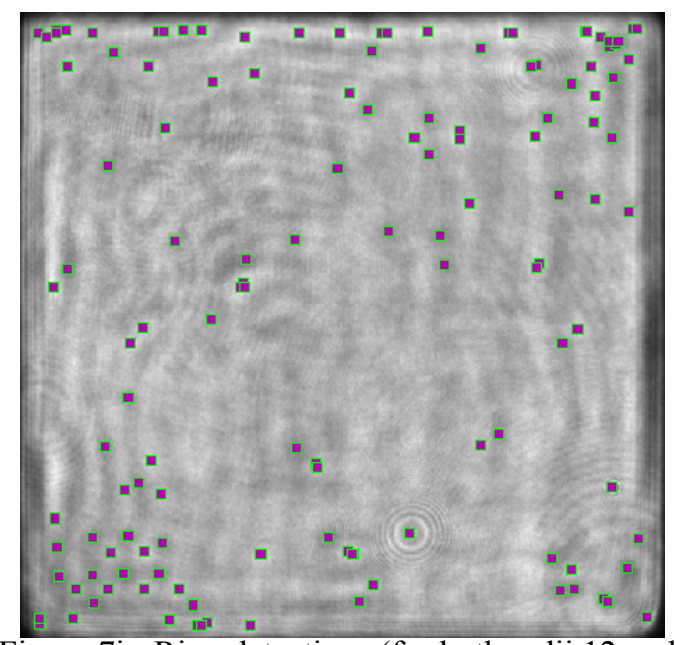

Figure 7j - Ring detections (for both radii 12 and 26) for example test image 1 before false alarm mitigation.

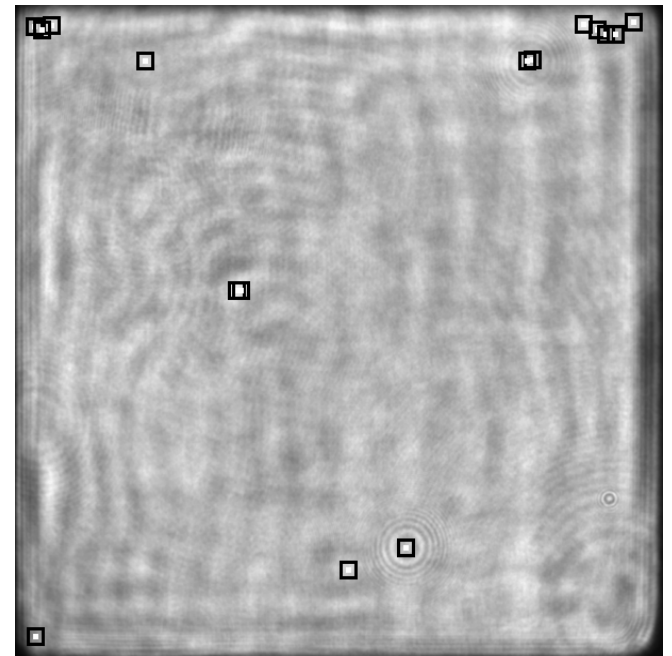

Figure $7 \mathrm{~g}-$ Initial ring detections for radius $=26$.

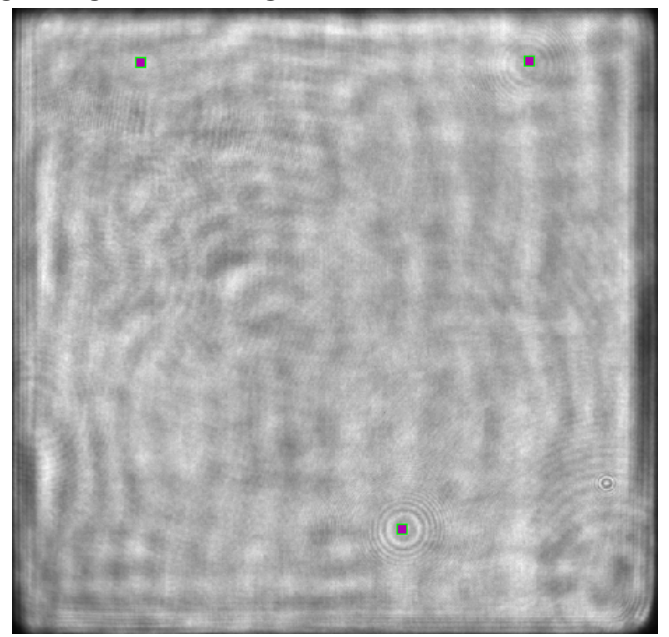

Figure 7i - Ring detections (radius=26) for example test image 1 after false alarm mitigation.

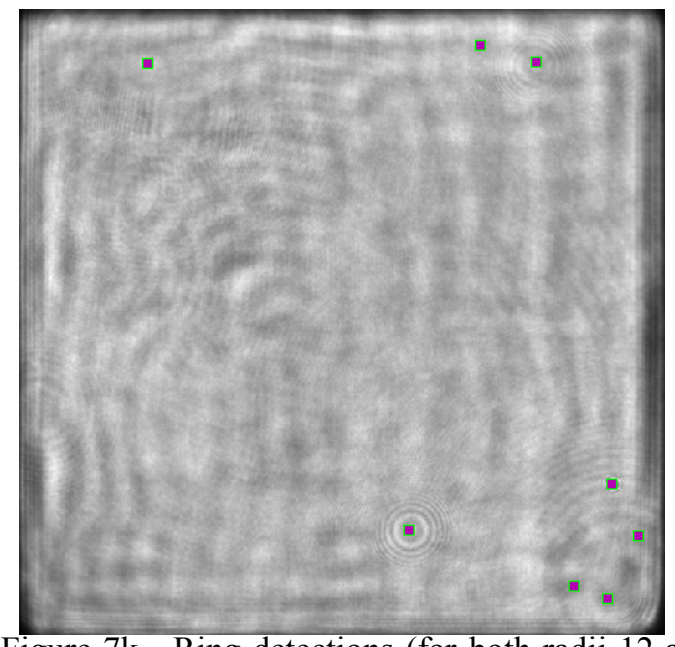

Figure 7k - Ring detections (for both radii 12 and 26) for example test image 1 after false alarm mitigation. 


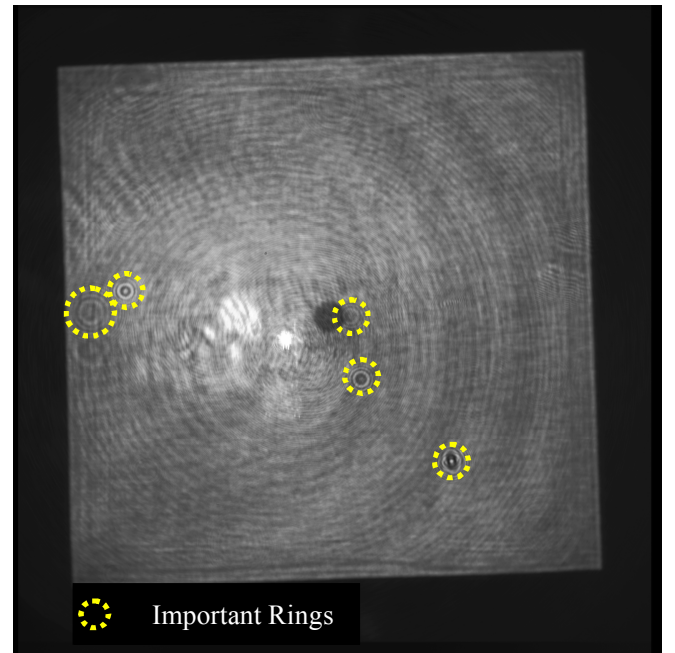

Figure $8 \mathrm{a}$ - Test image 2 (4200x4210 pixels).

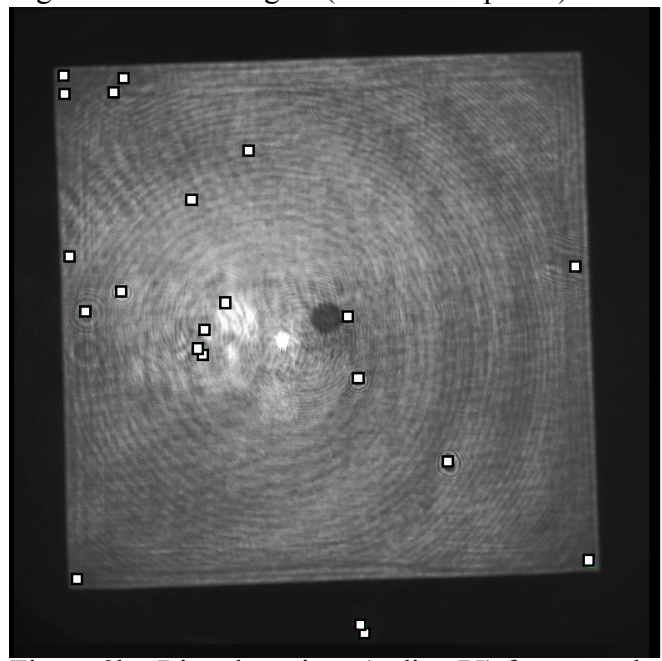

Figure $8 \mathrm{~b}$ - Ring detections (radius 75) for example test image 2 before false alarm mitigation.

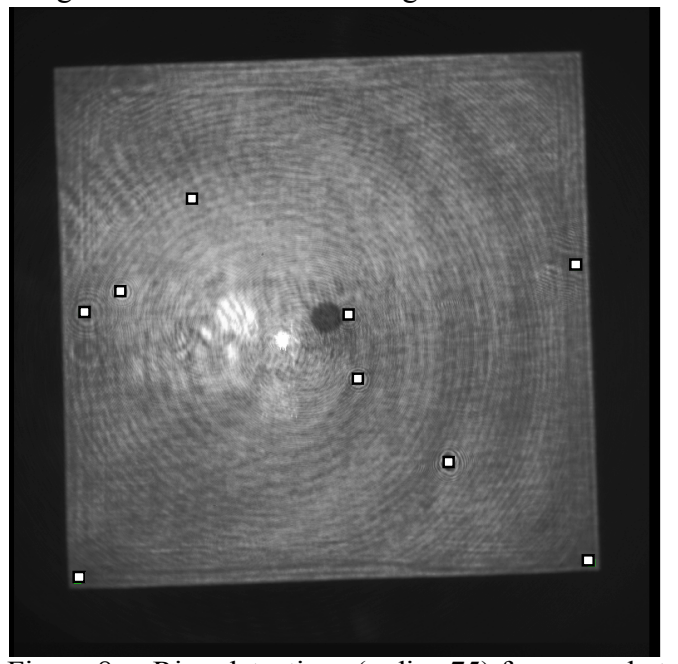

Figure 8c - Ring detections (radius 75) for example test image 2 after false alarm mitigation.
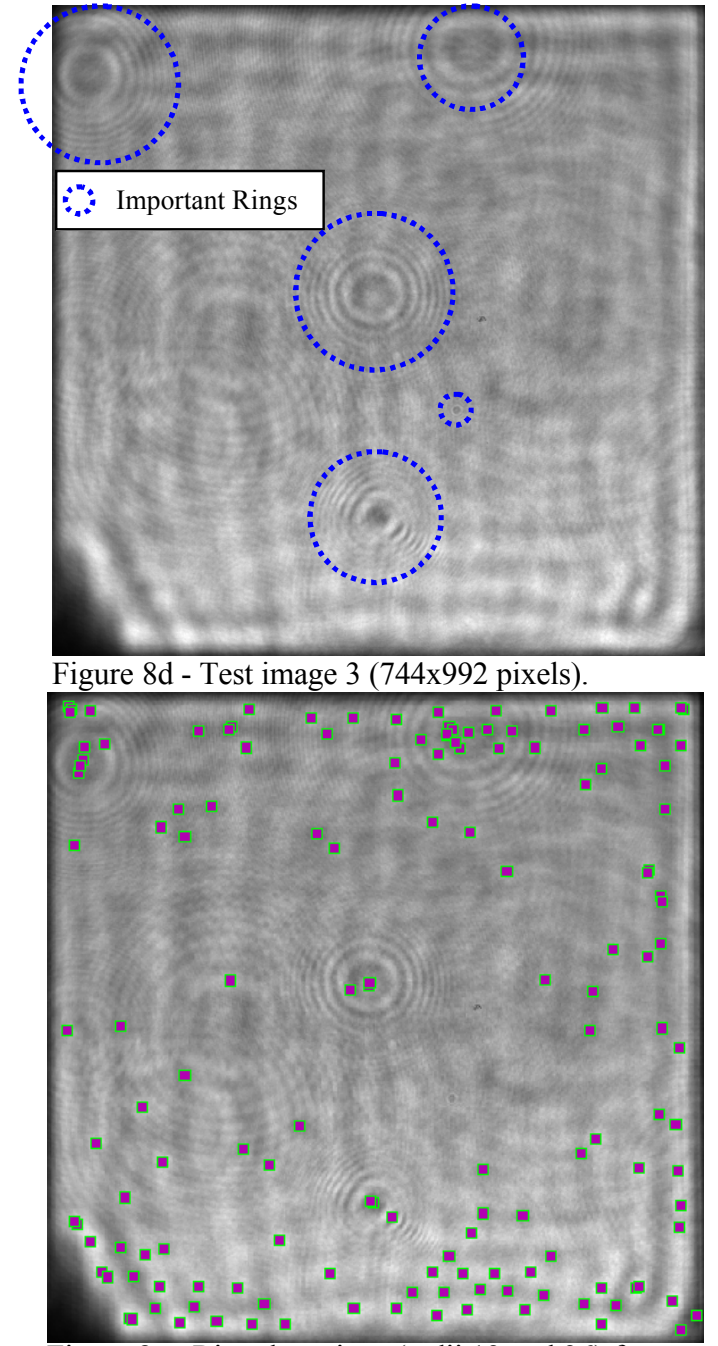

Figure 8e - Ring detections (radii 12 and 26) for example test image 3 before false alarm mitigation.

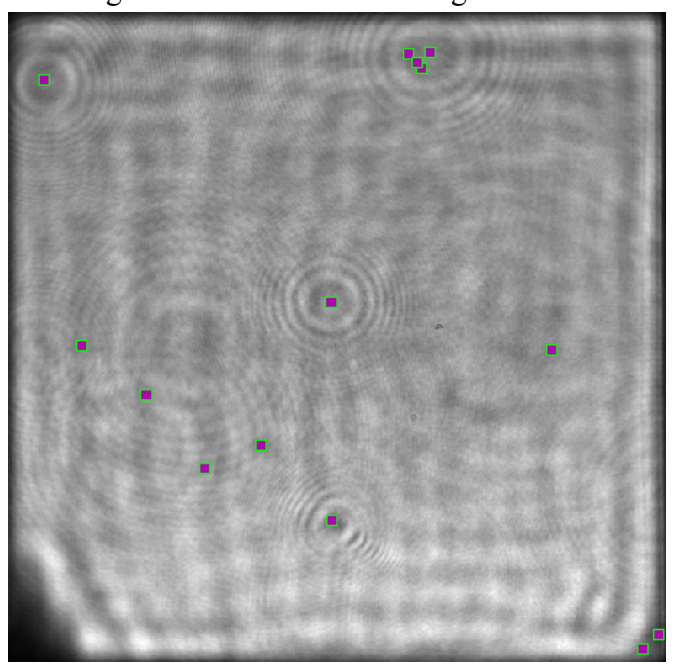

Figure 8f - Ring detections (radii 12 and 26) for example test image 3 after false alarm mitigation. 
Figures 8a-c show results of our algorithm on a 4200x4210 test image with five sharp rings (circled in dotted yellow) and two larger fuzzy rings. Notice that this image is darker and larger than the previous example test image. In this example, we have only used one luminance disk model with radius $=75$. Figure $8 \mathrm{~b}$ shows the initial ring detections followed by the final ring detections after false alarm mitigation in Figure 8c. The algorithm is able to detect all the important rings while returning only four false positives, demonstrating that our approach also works for larger images taken under different lighting conditions.

Figures 8d-f are images from another sample test image with lots of interfering ripples from large unimportant rings. This image has five important rings (circled in dotted blue) and several very large interfering rings. Using luminance disk radii of 12 and 26, the algorithm was not able to detect the tiny ring which has a radius of 5 pixels. When we used a disk radius of 8 , the small ring was detected; however, the number of false positives also detected was quite high. The four other important large rings are detected even when two of them are partially overlapped by larger unimportant rings. If we were interested in also finding these larger rings, we would need to use luminance disk radii much larger than 26 pixels. The overlapping rings have caused some false alarms to appear in places where their overlap cause small bight spots that look like the centers of real rings.

From these example test images and from others not pictured our algorithm is able to find most rings with a low false positive rate. There are, however, several cases where our algorithm has difficulty. First, tiny rings with radii less than $1 \%$ of the image width are difficult to detect without also detecting a lot of false positives. Both the match similarity value and ring fitness value for these small rings are not much different than those of the false positives. Second, the use of smaller luminance disk radii results in much more false detection. Therefore, when using smaller luminance disk radii, it is important to set the match similarity threshold at higher values to reduce the number of false detections. Third, rings cut off by the edge of the image are difficult to detect because part of the ring is missing which leads to lower similarity scores, and the edges of the image cause the algorithm to skew the placement of the detected ring centers. Finally, when there are two important rings that overlap by more than $50 \%$, the false alarm mitigation procedure will fail because the measured intensity curve will not well match the theoretical diffraction ring intensity curve.

\section{Summary and Topics for Future Research}

We have presented an approach for automatically detecting diffraction ring patterns in downstream optic images caused by an upstream scattering site. The approach uses GDM, an efficient and robust pattern matching tool, to find ring patterns by correlating their gradient directions with that of a luminance disk template. As a first stage, GDM quickly computes match similarity values for all pixels in the image. Similarity thresholding followed by local maxima finding produces an initial set of ring detections which are then sorted by how well the theoretical diffraction ring pattern fits them. The best fit ring detections and their corresponding defect size and distance estimates are the final results. Representative examples of our algorithm tested on a few images show that the algorithm is capable of detecting most rings of importance, while outputting a small number of false positive detections.

The two main topics for further study involve further reducing the number of false alarms for our ring detector, and integrating this ring detector with the defect detector. One way to further reduce the false detections is to incorporate ring detections gathered from images of other optics in the same beamline. The algorithm can rule out ring detections that do not also have ring detections in the same location on neighboring optic images. A related method involves comparing images of the same optic over time and ruling out ring detections that are not consistent through time. The other main area for further investigation is the integration of this indirect approach of finding rings with the direct approach that detects the defects themselves. Evidence of diffraction ring patterns can be used to corroborate defect detections and vice versa. 


\section{References}

1. L. M. Kegelmeyer. S.A. Daveler, J.A. Liebman, A. Jury, S.W. Ferguson, T. Vetsch, C. Orth, E. Bliss, T. Salmon, "NIF Optics Inspection (OI) Overview" CASIS Workshop Proceedings, November 14, 2003. Livermore, CA, UCRL-ABS-155663 p. 40.

2. C. M. G. Heffels, D. Heitzmann, E. D. Hirleman, B. Scarlett, "The Use of Azimuthal Intensity Variations in Diffraction Patterns for Particle Shape Characterization,” Part. Part. Syst. Charact. Vol. 11, 1994, pp. 194-199.

3. H. Wang, R. Valdivia-Hernandez, "Laser Scanner and Diffraction Pattern Detection: A Novel Concept for Dynamic Guaging of Fine Wires," Meas. Sci. Technol. Vol. 6, 1995, pp. 452-457.

4. D. W. Paglieroni, W. G. Eppler, D. N. Poland, "Phase Sensitive Cueing for 3D Objects in Overhead Images," Defense \& Security Symposium, Proc. SPIE Vol. 5809, Orlando, Fl, March 2005.

5. D. W. Paglieroni and W. G. Eppler, "Resolution Analysis for Gradient Direction Matching of Object Model Edges to Overhead Images", IEEE Trans. PAMI, November 2005, in review.

6. J. L. Horner, P. D. Gianino, "Phase-only matched filtering," Applied Optics, Vol. 23, No. 6., March 15, 1984, pp $812-816$.

7. A. Awwal, J. Candy, C. Haynam, C. Widmayer, E. Bliss, and S. Burkhart, "Accurate position sensing of defocused beams using simulated beam templates," Photonic Devices and Algorithms for Computing VI, Proc. SPIE Vol. 5556 (SPIE, Bellingham, WA) 2004, pp. 234-242.

8. H. D. Tagare, R. J. P. deFigueiredo, "On the Localization Performance Measure and Optimal Edge Detection," IEEE Trans. on Pattern Analysis and Machine Intelligence, Vol. 12, No. 12, December 1990, pp. 1186-1190.

9. P. W. Verbeek and L. J. van Vliet, "On the Location Error of Curved Edges in Low-Pass Filtered 2-D and 3-D Images," IEEE Trans. on Pattern Analysis and Machine Intelligence, Vol. 16, No. 7, July 1994, pp. 726-733.

10. I. E. Sobel, "Camera Models and Machine Perception," Ph.D. dissertation, Stanford University, Palo Alto, CA, 1970. 